\title{
Stress-dependent solute energetics in W-Re alloys from first-principles calculations
}

\author{
M. Z. Hossain ${ }^{\text {a }}$, Jaime Marian ${ }^{\mathrm{b}}$ \\ ${ }^{a}$ Division of Engineering and Applied Science, California Institute of Technology, Pasadena, CA 91125 \\ ${ }^{b}$ Lawrence Livermore National Laboratory, Livermore, CA 94551
}

\begin{abstract}
We present a systematic study of Re solute transport energetics in W using density functional theory calculations. The study focuses on substitutional solute diffusion in the presence of dislocation strain fields as a first step toward capturing the essential physics of solid solution hardening/softening in W-Re alloys. We calculate the heat of solution, the vacancy formation energy, and the solute migration energy as a function of both hydrostatic and shear strains. Our results show that the vacancy formation energy scales with hydrostatic deformation, whereas it decreases with increasing shear strain. The migration energy decreases with hydrostatic deformation, whereas it displays pathlength dependent behavior under shear deformation. In addition, we compute the binding energies of a Re solute atom to the cores of $1 / 2\langle 111\rangle$ screw and edge dislocations, and find the binding energy to be highest in the tensile lobe of the edge core. Finally, we obtain the dilatational stress due to a solute atom as a function of distance. Our calculations are then used to parameterize the jump rate of Re atoms in $\mathrm{W}$ as a function of the underlying stress state.
\end{abstract}

\section{Introduction}

As a refractory transition metal from the VI-A group, tungsten (W) is an important technological material with many attractive properties for functional and structural applications. However, $\mathrm{W}$ displays very low fracture toughness at low temperature, characterized by brittle trans and intergranular failure, which severely restricts its use as a structural material (Zinkle and Ghoniem, 2011).

$\mathrm{W}$ is typically alloyed with Re to increase low temperature ductility and improve high temperature strength and plasticity (Lassner and Schubert, 1999). As a consequence, W-Re alloys are used industrially in numerous applications. It is believed that Re stabilizes the grain structure in polycrystalline W-Re systems, increasing toughness and ductility. As well, recent calculations have shown that the Peierls stress of screw dislocations decreases in concentrated W-Re solid solutions, facilitating glide and plasticity (Romaner et al., 2010; Wurster et al., 2010, 2011; Li et al., 2012; Samolyuk et al., 2013). In any case, solid solution strengthening in W-Re alloys is governed microscopically by the compounded effects of solute diffusion under dislocation stress fields and dislocation glide in a solute field. Both of these processes are inherently atomistic, and, thus, methods capable of atomic-scale resolution and of capturing the alloy chemistry are required to study them. Electronic structure methods based on density functional theory (DFT) are ideally suited for this purpose. However, these methods involve substantial computational challenges, due to the necessity of dealing with the long-range elastic fields and long timescale phenomena - both of which are needed to understand solid solution hardening, but are beyond the scope of any first-principles method alone. Nevertheless, DFT results can act as the physical foundation upon which several multiscale schemes can be built and parameterized.

As is the case for most body-centered cubic (bcc) metals, the interest lies in simulating the coevolution of thermally-activated screw dislocation motion and solute diffusion. These are optimally 
simulated using stochastic methods such as the kinetic Monte Carlo (kMC) method (Voter, 2007), where rare-event rates are defined from the fundamental atomistic energetics. Therefore, determining the energetics accurately from DFT calculations to build a multiscale framework and describing the underlying mechanisms from a first-principles perspective constitute the focus of this paper.

The paper is organized as follows. In the first half, we study the dependence on stress of the diffusivity of Re atoms in bulk W. In the second half, we quantify the effect of solute atoms on dislocation kink-pair nucleation and segment pinning. The paper comprises four sections: in Section 2 we discuss the theoretical context of the study and outline the construction and verification of the different elements of the DFT calculations; in Section 3, results are provided for vacancy formation and migration energies in $\mathrm{W}$, both in nominal conditions and under different applied stresses; in Section 4, we quantify the stress field due to solute atoms, and compute their binding energies to dislocation cores. We finish in Section 6 with a discussion of our main findings and the conclusions.

\section{Computational model}

\subsection{Theoretical background}

In kMC simulations, the probability per unit time of a solute jump into a neighboring vacant site is given by the following expression:

$$
J=J_{0} \exp \left[-\frac{\Delta H_{v}}{k T}\right] \exp \left[-\frac{\Delta H_{m}}{k T}\right]
$$

where $J_{0}$ is a prefactor, and $\Delta H_{v}$ and $\Delta H_{m}$ are the vacancy formation and solute migration enthalpies. $J_{0}=z \nu_{0}$ includes an atomic coordination factor reflecting the number of possible nearest neighbor sites $(z=1 / 8$ for bcc $\mathrm{W})$ and an attempt frequency $\nu_{0}$, typically taken as the Debye frequency. The first Boltzmann factor in eq. 1 represents the equilibrium concentration of vacancies in the system, whereas the second one reflects the probability for a solute atom to exchange positions with a vacancy. Both the formation and migration enthalpies can be broken into their constituent internal energy $E$ and mechanical work $W_{m}$ (Hinterberg et al., 2013):

$$
\Delta H=\Delta E-W_{m}=\Delta E-\boldsymbol{\sigma}: \boldsymbol{V}^{a}
$$

where $\Delta E$ is the internal energy change at zero strain, $\sigma$ is the stress tensor, $\boldsymbol{V}^{a}$ is the activation volume tensor, and (:) denotes inner product between the tensors. The stress tensor $\boldsymbol{\sigma}$ may contain contributions from multiple sources such as externally applied stresses or dislocation stress fields. The activation volume tensor components can be computed from the derivatives of total energy with respect to the applied stress:

$$
V_{i j}^{a}=\frac{\partial E}{\partial \sigma_{i j}} .
$$

Although we are concerned mainly with the stress fields arising from screw and edge dislocations, we decompose the stress tensor into its spherical and deviatoric components, without explicitly considering their origin. This allows the enthalpies $\Delta H_{v}$ and $\Delta H_{m}$ to be coupled to any stress field, originating or not from dislocation lines. For simplicity, in DFT calculations it is preferable to work with strain fields imparted on the simulation boxes. Consequently, strain dependent variables can be computed directly from DFT simulations by (i) deforming the lattice according to a desired strain $\varepsilon$, (ii) computing the energetic variation as a function the deformed state, and (iii) obtaining a relation

$$
\Delta H=f(\varepsilon)
$$

from optimal fits to the data points describing the strain-dependent energetics. Because $\mathrm{W}$ is isotropic elastic, in the limit of small deformations we can trivially convert the strain into stress via the relation:

$$
\sigma_{i j}=2 \mu \varepsilon_{i j}+\frac{3 \nu B}{1+\nu} \varepsilon_{k k} \delta_{i j}
$$


where $\mu, \nu$, and $B$ are, respectively, the shear modulus, Poisson's ration and the bulk modulus. For clarity, in the foregoing, we use the following notation to refer to the strain components:

- $\epsilon=\operatorname{Tr}\left(\varepsilon_{k k}\right) \approx 3 \varepsilon$ is the volumetric strain (with $\varepsilon$ being a generic linear strain).

- $\gamma=2 \varepsilon_{i j}$, with $i \neq j$, is the engineering shear strain.

In the following sections, we describe the DFT methodology and the computational details employed to carry out the calculations, and discuss the results in detail.

\subsection{Ab initio calculations}

The ab initio calculations are performed using the SIESTA (Soler et al., 2002) code, which carries out self-consistent density functional theory calculations with norm conserving pseudopotentials and a flexible linear combination of numerical atomic orbital basis. The core electrons are replaced by Troullier-Martins pseudopotentials (Troullier and Martins, 1991) and the valence electrons are described by a Double-Zeta Polarized (DZP) orbital basis set (Soler et al., 2002). For the exchange-correlation part of the energy functional, we used the local density approximation (LDA) parameterized by Ceperley and Alder. The convergence of the energy is achieved for a plane-wave cutoff of 300 Ry. Throughout the calculations, both local and volumetric relaxations are carried out using the Broyden conjugate gradient algorithm and a force tolerance of $0.01 \mathrm{eV} / \AA$. Convergence of the results is checked against different $k$-mesh densities depending on the systems studied.

To construct a given strain state, the lattice vectors of the supercell are determined using the following relation:

$$
\boldsymbol{a}^{\prime}=(\boldsymbol{I}+\boldsymbol{\varepsilon}) \boldsymbol{a},
$$

where $\boldsymbol{a}^{\prime}, \boldsymbol{I}, \boldsymbol{\varepsilon}, \boldsymbol{a}$ are the deformed lattice vectors, identity tensor, strain tensor, and the undeformed lattice vectors, respectively. The hydrostatic and deviatoric terms of the sum $(\boldsymbol{I}+\boldsymbol{\varepsilon})$ can be written as:

$$
\left[\begin{array}{ccc}
1 \pm \varepsilon & 0 & 0 \\
0 & 1 \pm \varepsilon & 0 \\
0 & 0 & 1 \pm \varepsilon
\end{array}\right] \text { and }\left[\begin{array}{ccc}
1 & \gamma / 2 & 0 \\
\gamma / 2 & 1 & 0 \\
0 & 0 & 1
\end{array}\right] \text {, respectively. }
$$

The notation employed here is that tension (compression) is represented by $\varepsilon>(<) 0$. The undeformed lattice vectors are $\boldsymbol{a}_{1}=\left(a_{0}, 0,0\right), \boldsymbol{a}_{2}=\left(0, a_{0}, 0\right)$, and $\boldsymbol{a}_{3}=\left(0,0, a_{0}\right)$ with $a_{0}=3.1336 \AA$ (cf. Section 2.3.1). For volume conserving shear deformations, the lattice vectors of the deformed lattice are: $\boldsymbol{a}_{1}^{\prime}=\left(a_{0}, 0,0\right), \boldsymbol{a}_{2}^{\prime}=\left(a_{0} \tan \theta, a_{0}, 0\right)$, and $\boldsymbol{a}_{3}^{\prime}=\left(0,0, a_{0}\right)$, where $\theta=\tan ^{-1} \gamma$ is the shear angle.

\subsection{Generation and validation of the pseudopotential}

To generate pseudopotentials for tungsten, the electronic configuration of $\mathrm{W}$ is decomposed into two sets of electronic orbitals: (a) the orbitals contributing as core electrons, and (b) the orbitals contributing as valance electrons. The core of the pseudo-atom consists of $1 s^{2}, 2 s^{2}, 2 p^{6}, 3 s^{2}, 3 p^{6}$, $4 s^{2}, 3 d^{10}, 4 p^{6}, 5 s^{2}, 4 d^{10}, 5 p^{6}$ and $4 f^{14}$ electronics states, and the valence shell is constructed with the $6 s^{2}, 6 p^{0}, 5 d^{4}$, and $5 f^{0}$ electronic states. The cutoff radii $r_{c}$ for the valence orbitals that contains all the nodes formed by the wiggling of all-electron wavefunctions near the atomic-core are chosen to be $2.70,2.77,2.51$ and 2.51 bohr for the $6 s^{2}, 6 p^{0}, 5 d^{4}$, and $5 f^{0}$, respectively, such that the pseudo-wavefunctions represent all-electron wave functions accurately for $r \geq r_{c}$, as depicted in Fig. 1. The potential was tested against different choices of the valence configuration, such as $\left(6 s^{2}, 5 d^{4}\right),\left(6 s^{2}, 6 p^{1}, 6 d^{3}\right),\left(6 s^{2}, 6 p^{2}, 5 d^{2}\right),\left(6 s^{1}, 6 p^{1}, 5 d^{4}\right)$, and $\left(6 s^{2}, 6 p^{0}, 5 d^{3}, 6 f^{1}\right)$. The energy difference between all-electron calculations and pseudopotential calculations falls below $0.1 \mathrm{mRy}$ for each of this electronic configurations. 

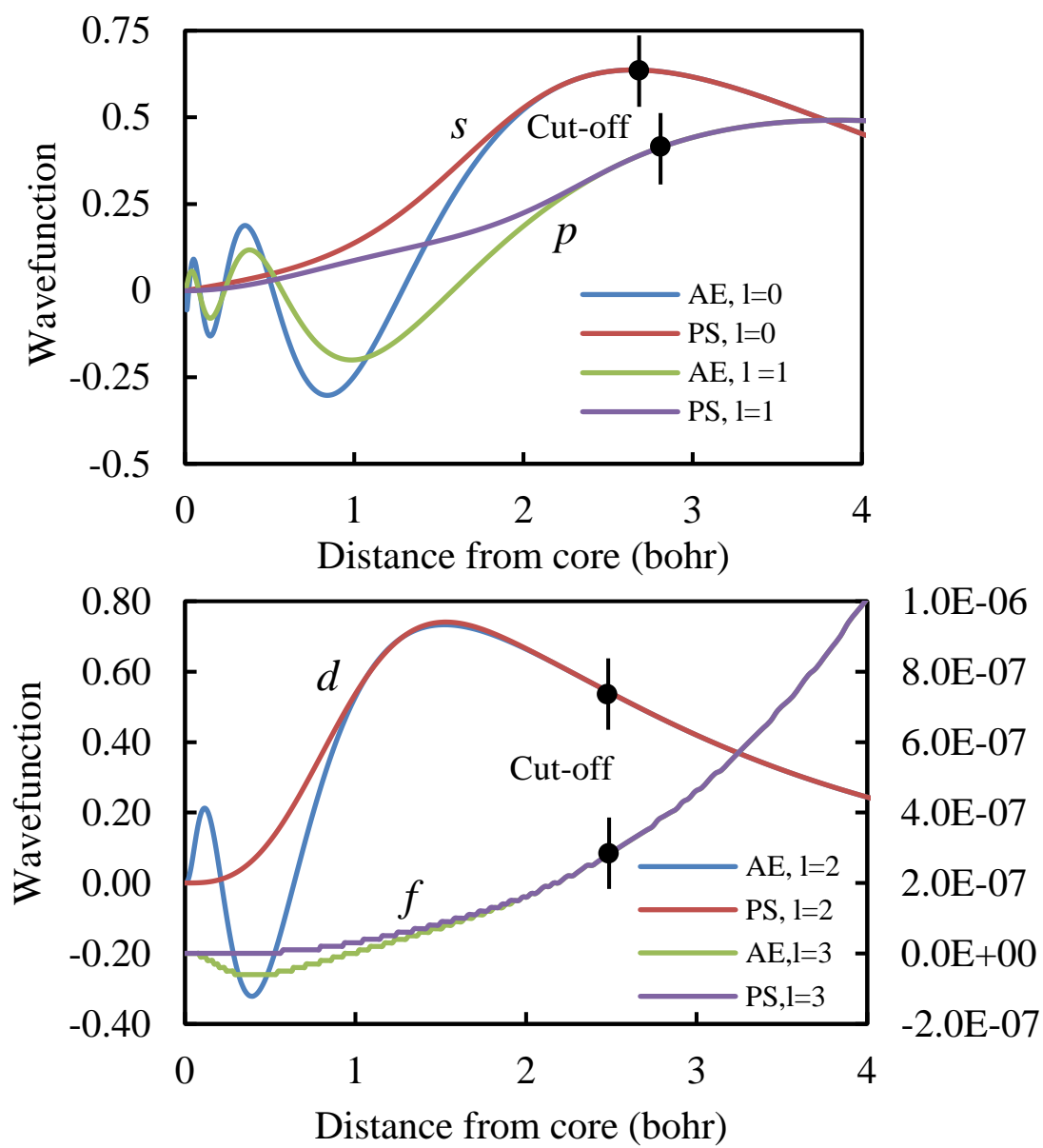

Figure 1: Comparison of valence electron energies obtained with an actual atom (containing all electrons and actual potentials) and the pseudo-atom containing the pseudopotential. The respective cut-off electron orbitals for the valence states are shown in the plots.

Nevertheless, the true validation of the pseudopotential comes from its ability to produce accurate physical parameters. As a perfectly isotropic elastic material, only two elastic parameters are sufficient to define tungsten's elasticity. We choose to calculate the bulk modulus $(B)$ and Poisson's ratio $(\nu)$ and obtain other relevant elastic parameters using linear elasticity relations.

\subsubsection{Elastic moduli, and lattice constant}

Structural parameters are computed with a 2 -atom unit cell and a $k$-mesh of $24 \times 24 \times 24$, which is sufficiently large for convergence of the total energy. The bulk modulus and lattice constant are then obtained by fitting the Murnaghan equation of state, eq. 4, to the variation of total energy of a crystalline $\mathrm{W}$ lattice with respect to the volumetric changes of the lattice, as shown in Fig. 2(a).

$$
E(V)=E_{0}+\frac{B_{0} V}{B_{0}^{\prime}}\left[\frac{\left(V_{0} / V\right)^{B_{0}^{\prime}}}{B_{0}^{\prime}-1}+1\right]-\frac{B_{0} V_{0}}{B_{0}^{\prime}-1}
$$

Here $E, V, B_{0}, E_{0}$ and $B_{0}^{\prime}$ denote the energy, volume, bulk modulus, cohesive energy, equilibrium volume, and the gradient of the bulk modulus with respect to pressure, respectively. The fit yields: $B_{0}=308.8 \mathrm{GPa}, V_{0}=30.83 \AA^{3}, B_{0}^{\prime}=3.69, a_{0}=V_{0}^{1 / 3}=3.1356 \AA$. 


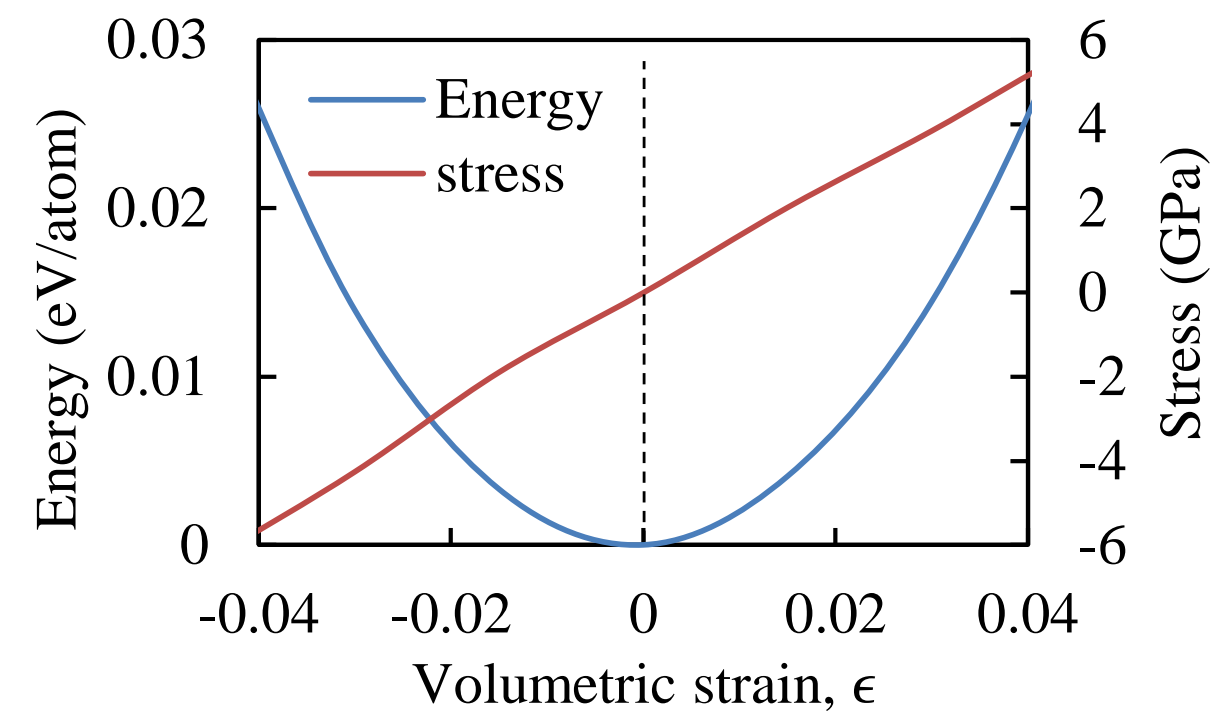

Figure 2: Variation of total energy and stress as a function of volumetric strain. The primary and the secondary axes represent energy and stress, respectively.

To compute Poisson's ratio, the lattice is deformed along the [100] direction by $2 \%$ tensile strain, and the lateral contraction is measured by taking the volumetric variation of the lattice energy with respect to a change in lattice dimension only in such direction. Fitting a second-order polynomial to the resulting energy versus transverse lattice parameter curve and taking its first derivative equal to zero, Poisson's ratio is obtained to be 0.276 . The Young's modulus $E$ and the shear modulus $\mu$ are computed using the equations of linear elasticity for isotropic solids: $E=3 B(1-2 \nu)=415 \mathrm{GPa}$, and $\mu=\frac{3 B(1-2 \nu)}{2(1+\nu)}=163.0$ GPa. A comparison, shown in Table 1, of the DFT results with available experimental values indicates reasonable agreements for the structural parameters considered.

Table 1: The elastic moduli are all given in GPa, while the lattice parameter is expressed in $\AA$.

\begin{tabular}{|c|c|c|c|}
\hline constant & this work & experiment & difference [\%] \\
\hline$B$ & 309 & 314 & 1.6 \\
$\mu$ & 163 & 160 & 1.8 \\
$E$ & 415 & 411 & 0.9 \\
$\nu$ & 0.276 & 0.28 & -1.4 \\
$a_{0}$ & 3.1336 & 3.1583 & -0.7 \\
\hline
\end{tabular}

\section{Strain-dependent solute energetics}

By separating the applied strain into volumetric and shear components, one can use superposition to study the effect of arbitrary strain fields, including those of dislocations. Next, we study the dependence on strain of the energetics of solute atoms and vacancies in deformed lattices. 


\subsection{Enthalpy of solution as a function of volumetric strain}

The enthalpy of solution $\Delta H$ is defined as the extra energy required to insert a Re atom in a $\mathrm{W}$ matrix. Since W-Re are substitutional alloys, the enthalpy of solution $\Delta H$ can be expressed as:

$$
\Delta H_{\mathrm{Re}}(\epsilon)=E(N-1,1 ; \epsilon)-\frac{N-1}{N} E(N, 0 ; \epsilon)-\frac{1}{M} E(0, M ; \epsilon)
$$

where $E(N, M, \epsilon)$ is the energy of a superlattice containing $N$ tungsten atoms and $M$ rhenium atoms at volumetric strain $\epsilon$ and hence $E(0, M ; \epsilon)$ is the energy of a system of $M$ Re atoms. For calculating $E(0, M ; \epsilon)$ any stable solid phase of Re can be considered, since a single Re atom has no atomic structure of its own inside $\mathrm{W}$. This makes the enthalpy of solution, $\Delta H_{\mathrm{Re}}$, a measure of energy relative to the solid phase considered. Fortunately, even for unstrained Re, the enthalpy of solution has been shown to be only weakly dependent on the choice of the crystal phase (Wierzbowska and Fleszar, 2011).

For the matrix W crystal, a $4 a_{0} \times 4 a_{0} \times 4 a_{0}$ supercell with $N=128$ atoms and a $k$-mesh of $8 \times 8 \times 8$ are used. For the Re system, we have chosen one fcc primitive cell containing $M=4$ atoms with 2192 mesh points in $k$-space. The equilibrium lattice constant obtained in the calculation for Re is $3.8759 \AA$, which is deformed hydrostatically within $\pm 8 \%$ to determine $E(0, M ; \epsilon)$.

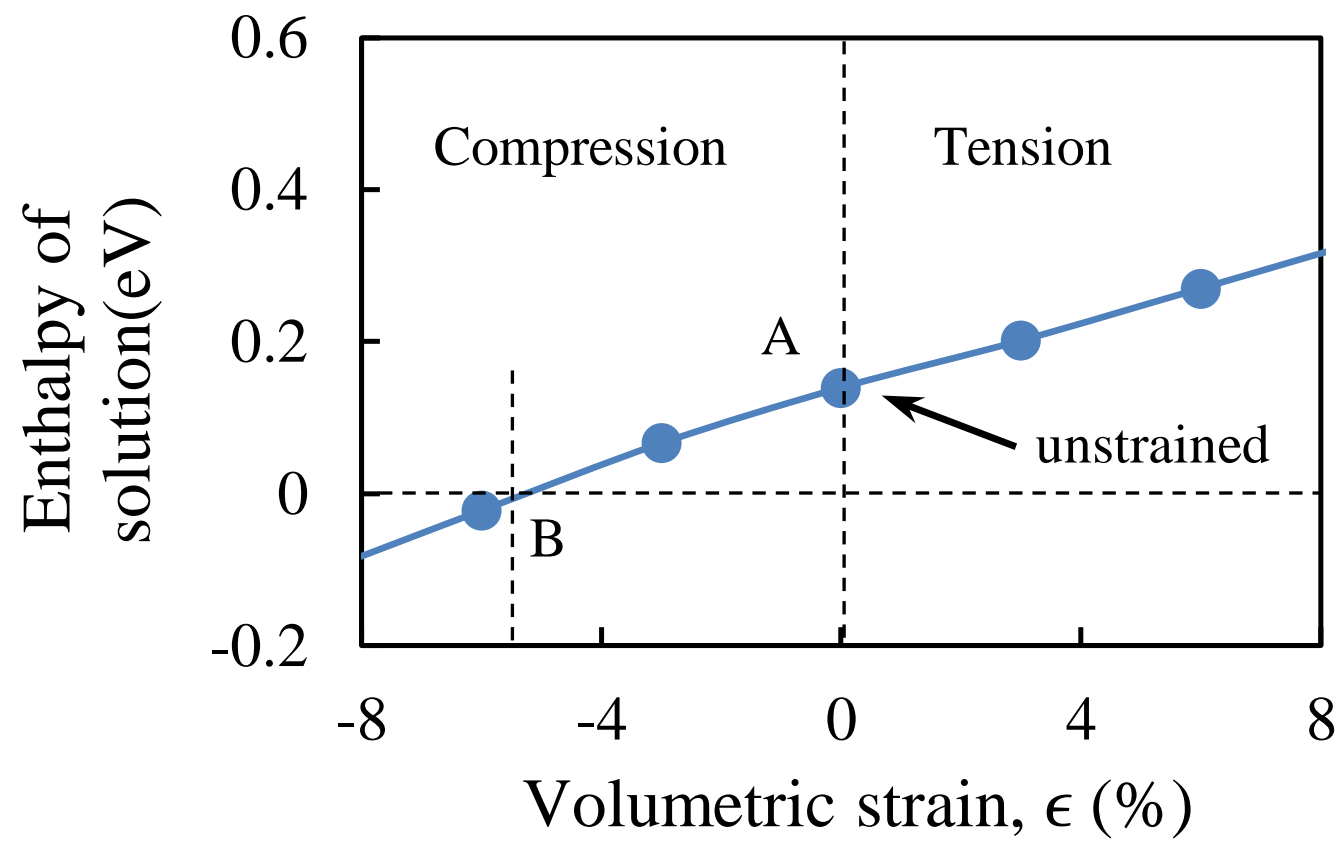

Figure 3: Enthalpy of solution of a Re atom in bcc W. The points labeled 'A' and 'B' mark the enthalpies at $\varepsilon=0$ and $\Delta H<0$, respectively.

Using eq. 5, the enthalpy of solution is then computed as a function of volumetric strain and displayed in Fig. 3. A linear fit, accurate to first order, to the data points in the figure yields:

$$
\Delta H_{\operatorname{Re}}(\epsilon)=0.024 \epsilon+0.1395
$$

The independent term in the above equation defines the heat of solution $\Delta H_{\mathrm{Re}}(0)$ to be $=0.1395 \mathrm{eV}$ per atom, or $3.24 \mathrm{kcal}$ per mol, indicating the enthalpy of a Re atom in an unstrained W environment. At $\epsilon=-6 \%$ or $\sigma_{h}=B \epsilon=-18.48 \mathrm{GPa}$, the enthalpy of solution becomes zero and the Re atom becomes an ideal solute, absorbing or releasing no energy to the host lattice. 
For compressive strains larger than $6 \%$ (point ' $\mathrm{B}$ ' in Fig. 6), the heat of solution becomes negative, indicating that a bcc Re phase is energetically more favorable than the $\mathrm{W}$ bcc structure (Watson et al., 1988). By contrast, under tensile stress $\Delta H_{R e}(0)$ increases, reducing the solubility of Re in $\mathrm{W}$. This is an indication that substitution of Re in W creates a tensile field around the Re atom in unstrained conditions. This behavior is consistent with a symmetry preserving deformation (Zhou et al., 2012), as well as with what we find from the direct calculations discussed in Section 4.

\subsection{Vacancy formation energy as a function of volumetric strain}

The vacancy formation enthalpy $\Delta H_{\mathrm{v}}$ can be computed as:

$$
\Delta H_{\mathrm{v}}(\epsilon)=E(N-1,1 ; \epsilon)-\frac{N-1}{N} E(N, 0 ; \epsilon),
$$

where $E\left(N, N_{\mathrm{v}} ; \epsilon\right)$ represents the total energy of a lattice containing $N$ tungsten atoms and $N_{\mathrm{v}}$ vacancies under volumetric strain $\epsilon$. A vacancy defect creates local distortions that must be relaxed to find equilibrium configurations prior to computing the corresponding equilibrium energies. However, the effect of volume relaxation vanishes for supercells containing more than 2 unit cells along each of the three directions (Söderlind et al., 2000; Korzhavyi et al., 1999). In this work, a supercell containing $N=128$ atoms (or 4 unit cells along each of the three cartesian coordinate directions) was found to be sufficient to avoid any spurious effects arising from the periodic images.

Prior to studying the dependence of $\Delta H_{\mathrm{v}}$ on $\epsilon$, we consider the effect on the electronic density of the creation of an internal surface due to the formation of a vacancy. This results in corrections to the vacancy formation energy, particularly through the exchange-correlation part of the energy functional, that must be carefully considered (Carling et al., 2000; Mattsson et al., 2006; Nazarov et al., 2012). Assuming the correction to depend on the surface area of the vacancy and the bulk electron density (Mattsson et al., 2006; Delczeg et al., 2009), we provide an estimate of the intrinsic surface error on $\Delta H_{\mathrm{v}}$. We compute plane-averaged electron densities, as shown in Fig. 4, to find the maximum, minimum, and average electron densities in the bulk, and quantify the associated surface corrections using the following expression:

$$
\Delta H_{\mathrm{v}}^{\mathrm{corr}}=\Delta H_{\mathrm{v}}+4 \pi R^{2} \Delta \sigma\left(n_{e l}\right),
$$

where, $R, \Delta \sigma$, and $n_{e l}$ are the radius of the internal surface of the monovacancy, the energy correction per unit surface area, and the bulk electron density, respectively. $n_{e l}^{\max }($ bulk $)=0.391, n_{e l}^{\min }$ (bulk) $=0.385, n_{e l}^{\text {avg }}($ bulk $)=0.388 \AA^{-3}$. In the homogeneous non-interacting electron approximation, the electron density is $762 /\left(4 \times(3.1336)^{3}\right)=0.386 \AA^{-3}$. $R$ can be computed assuming that its ratio with a known value of $R$ for a reference material obtained using the homogeneous electron approximation scales with the ratio of lattice constants. Here we use values for Al (Mattsson and Mattsson, 2002): $R / R_{\mathrm{Al}}=a_{0} / a_{\mathrm{Al}}$, which, for $R_{\mathrm{Al}}=1.2 \AA$ and $a_{\mathrm{Al}}=4.03 \AA$, yields $R=0.933 \AA$.

Using the generic relation between electron density and surface energy correction per unit surface outlined in the literature (Carling et al., 2000; Mattsson and Mattsson, 2002; Mattsson et al., 2006), the energy correction, $\Delta \sigma$ is obtained approximately to be $0.005 \mathrm{eV} / \AA^{2}$ for $n_{e l}^{\min } \leq n_{e l} \leq n_{e l}^{\max }$ within the LDA approximation to the exchange-correlation energy. Taking the average bulk electron density, the resulting energy correction, $\Delta H_{\mathrm{v}}^{\text {corr }}$ becomes $\approx 0.05 \mathrm{eV}$, which can be considered independent of strain. For the unstrained lattice, $\Delta H_{\mathrm{v}}^{\text {corr }}=\Delta H_{\mathrm{v}}+\Delta H^{\text {corr }}=3.66+0.05=3.71 \mathrm{eV}$, which falls within the available experimental values 3.6-4.1 eV (Satta et al., 1998; Schultz and Ehrhart, 1991), and is in good agreement with available computational estimates, such as $3.64 \mathrm{eV}$ (Söderlind et al., 2000), obtained using DFT-LDA without surface correction.

\subsubsection{Dependence with hydrostatic deformation}

The variation of the normalized vacancy formation energy $\Delta \bar{H}_{\mathrm{v}}$ with respect to the applied hydrostatic strain is shown in Fig. 5 for $-0.25<\epsilon<0.1$. The maximum value of $\epsilon$ considered 


\section{(a)}

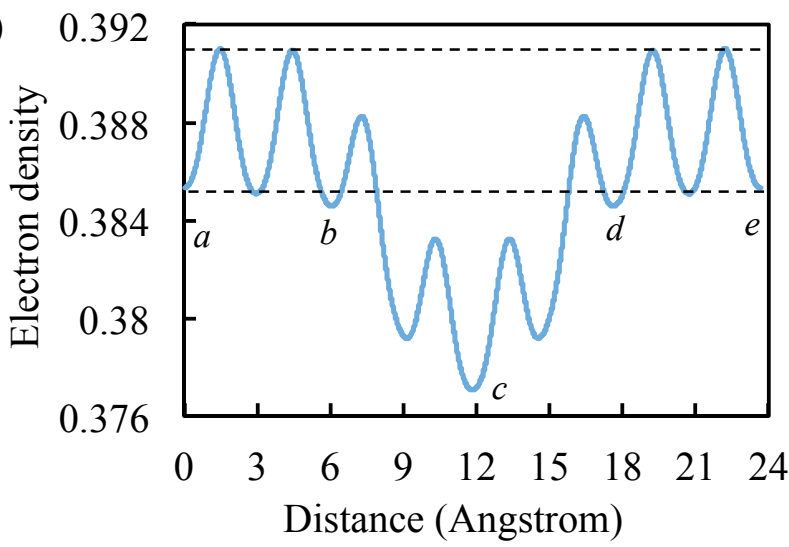

(b)

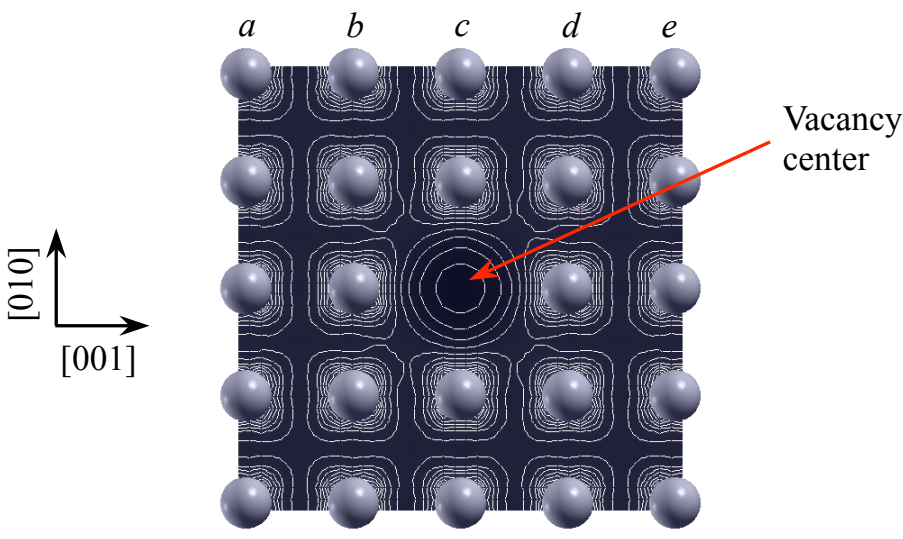

Figure 4: (a) Effect of a vacancy on the $\{001\}$-plane-averaged electron density variation across the vacancy and (b) isolines showing electron densities in the range $0 \leq n_{e l} \leq 0.20$ in the plane $4 a_{0}\langle 200\rangle$ passing through the vacancy, where $a_{0}$ is the lattice constant of $\mathrm{W}$. The vacancy is positioned at the center of the supercell. The plane averagedelectron densities on the planes marked by $a, b, c, d$, and $e$ are identified in (a). The maximum and minimum electron densities are marked by the dotted lines.

here (0.1) is established by the ideal tensile strength of $\mathrm{W}$, around $29 \mathrm{GPa}$ (Giusepponi and Celino, 2013). In this range, the curve is well fitted by a quadratic polynomial:

$$
\Delta \bar{H}_{\mathrm{v}}=-8.867 \epsilon^{2}+3.135 \epsilon+1.0
$$

For $\epsilon=0.1, \Delta H_{\mathrm{v}} \approx 1.2 \Delta H_{\mathrm{v}}(0)$. Under compressive strain, the vacancy formation energy decreases up to a value of $\epsilon \approx-20 \%$. Beyond that point, which corresponds to a pressure of 61.6 $\mathrm{GPa}$, the vacancy formation energy becomes negative, meaning that vacancies are spontaneously generated in the crystal. The two endpoints of the strain interval considered here are limits of ideal behavior. In practical situations, other more complex phenomena occur prior to achieving the ideal tensile limits of the material.

The trend observed in Fig. 5 can be ultimately explained by the effect that a vacancy has on the local electron density distribution. A vacancy creates a local depletion in the surrounding electron density -akin to having 'dangling' bonds in ionic or covalent materials- which draws neighboring atoms toward the vacant site to compensate this effect. Indeed, we have measured this tensile stress at $4.267 \times 10^{-3} \mathrm{eV} / \AA^{3}$ or $0.68 \mathrm{GPa}$ (by way of comparison, the insertion of a Re atom causes a tensile stress of $0.125 \mathrm{GPa}$ ). Compressing the lattice aids in balancing this tensile stress, decreasing the 


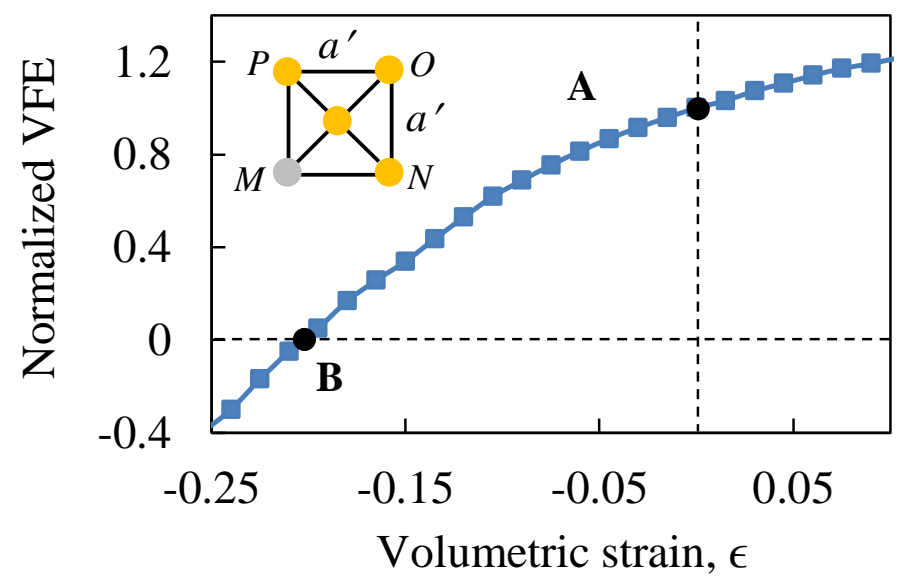

Figure 5: Normalized vacancy formation energy (VFE), $\Delta \bar{H}_{\mathrm{v}}$ as a function of volumetric strain. The positions marked by $A$ and $B$ denote, respectively, the points where $\Delta \bar{H}_{\mathrm{v}}=1, \Delta \bar{H}_{\mathrm{v}}=0$.

energy required to create the vacant site. Conversely, under tension, it takes more energy to form a vacancy because the applied stress and the local tensile stress are additive.

Although the number of dangling bonds per vacancy is fixed, the magnitude of applied deformation can alter their energetics. The atoms undergo local relaxation to minimize the elastic energy induced by the applied and local deformations. However, generally, the energetic alteration of the lattice due to the formation of a vacancy is dominated by the local relaxation, particularly for larger supercells, where volume relaxation hardly interferes with the local elastic field at the vacant site.

\subsubsection{Dependence with shear deformation}

Next, we compute the vacancy formation energy as a function of simple shear $\gamma$, which is applied from 0 to $3.5 \%$. The corresponding variation in normalized formation energy is plotted in Fig. 6, and the calculated data points are fitted to a cubic polynomial:

$$
\Delta \bar{H}_{\mathrm{v}}=-2978 \gamma^{3}+80 \gamma^{2}-2.35 \gamma+1.0
$$

Under shear deformation, some of the nearest neighbor bonds are put under tension (along the body diagonal $M O$ schematically shown in Fig. 6 and some under compression (body diagonal $P N$ ). The vacancy formation energy thus results from a combination of stretching and contraction of the bonds of the atoms surrounding the vacant site.

It is interesting to examine how an isochoric transformation results in changes for a volumetric defect such as a vacancy. At small shear strains, the energetic cost of extending and contracting bonds is comparable. This results in a slow decrease of $\Delta \bar{H}_{\mathrm{v}}$ up to approximately $\gamma<1.5 \%$. However, at larger strains, bond contraction requires more energy compared to bond stretching due to the increased electron-electron repulsion at distances smaller than the equilibrium distance. As a result, for $\gamma>1.5 \%$ the behavior of $\Delta \bar{H}_{\mathrm{v}}$ is governed by the compressive contribution, leading to a sharp decrease in the vacancy formation energy, as is evident from Fig. 6.

\subsection{Solute migration energy as a function of strain}

The migration energy barrier $\Delta H_{\mathrm{m}}$ is defined as the energy that an atom needs to overcome to jump into an available nearest vacant site from an occupied site. The migration process leads to a collective atomic relaxation process along the intermediate transitional states describing the reaction coordinate. Thus, the determination of $\Delta H_{\mathrm{m}}$ requires the determination of a suitable reaction pathway, defined by a set of constrained intermediate states, that constitutes a minimum 


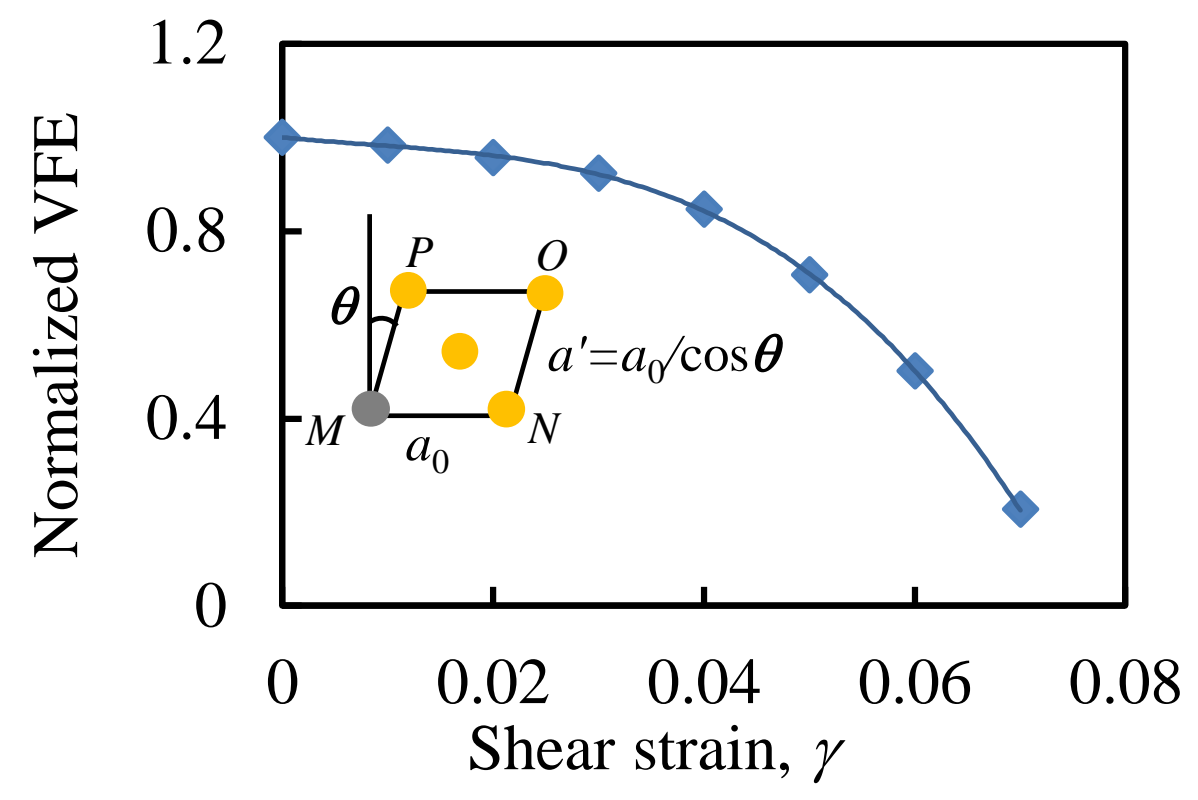

Figure 6: Normalized vacancy formation energy, $\Delta \bar{H}_{\mathrm{v}}$ under shear deformation. The corresponding deformed configurations are represented schematically, where $\gamma=\tan \theta$ is the net shear, $a^{\prime}$ is the deformed lattice parameter, and $\theta$ is the shear angle.

energy trajectory in configurational space. To generate these intermediate states, in this work we employ the drag method due to its simplicity in application and its reliability for a range of BCC systems (Fu et al., 2005; Weinberger et al., 2013). We apply this procedure to both W and Revacancy exchanges.

To this end, a $4 \times 4 \times 4$ supercell containing 128 lattice sites is constructed with a single vacancy positioned at the origin and a Re atom at a nearest neighbor position along the [111] direction. As shown in Fig. 7, nine intermediate replicas spaced uniformly are generated by linear interpolation between the initial and final states to compute the migration energy as a function of the reaction coordinate. The migration energy for Re and $\mathrm{W}$-vacancy exchanges is $1.57 \mathrm{eV}$ and $1.54 \mathrm{eV}$, respectively. The extra electron in Rhenium thus influences the migration barrier by $1.9 \%$.

For this calculation we have assumed that the surface energy corrections discussed in the previous section do not influence $\Delta H_{\mathrm{m}}$, i.e. the radius of the vacancy does not change along the migration path or under applied strain.

\subsubsection{Dependence with hydrostatic deformation}

For volumetric deformation, the normalized migration energy barrier and its dependence on supercell size and local strain relaxation are depicted in Fig. 8. Both compression and tension are found to reduce the migration energy barrier for Re. As the figure clearly shows, we find $\Delta H_{\mathrm{m}}$ to be very sensitive to the supercell size. We have considered $2 a_{0} \times 2 a_{0} \times 2 a_{0}$ and $4 a_{0} \times 4 a_{0} \times 4 a_{0}$ supercells and observed significantly different behaviors in each case. For the smaller system at a constant lattice volume, periodic images result in vacancy-vacancy interactions, affecting the ability of the atoms to relax locally around the vacancy site. In such case, enabling local relaxation reduces the energy only by a very small amount, causing the migration energy barrier to be artificially unaffected. On the other hand, for the larger supercell, the vacancy-vacancy interaction energy is negligible, resulting in larger differences between the unrelaxed and relaxed $\Delta H_{\mathrm{m}}$. In Fig. 8, the results for the $2 a_{0} \times 2 a_{0} \times 2 a_{0}$ system clearly display a linear behavior with strain that changes 


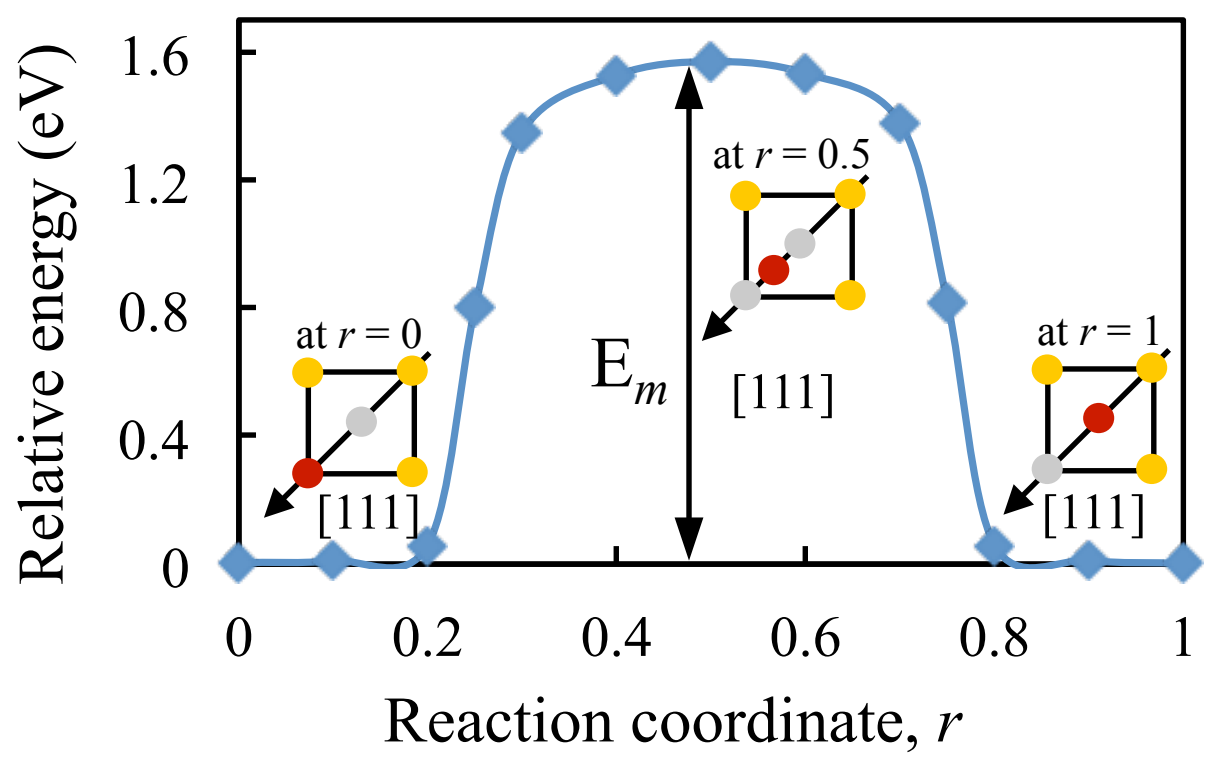

Figure 7: Migration energy barrier for Re in an unstrained W lattice. The relative positions of Re with respect to the $\mathrm{W}$ atoms and the vacancy site at three positions along the drag-coordinate are shown schematically, where $\mathrm{W}$ atoms, Re atoms and the vacancy are shown in yellow, red and gray, respectively.

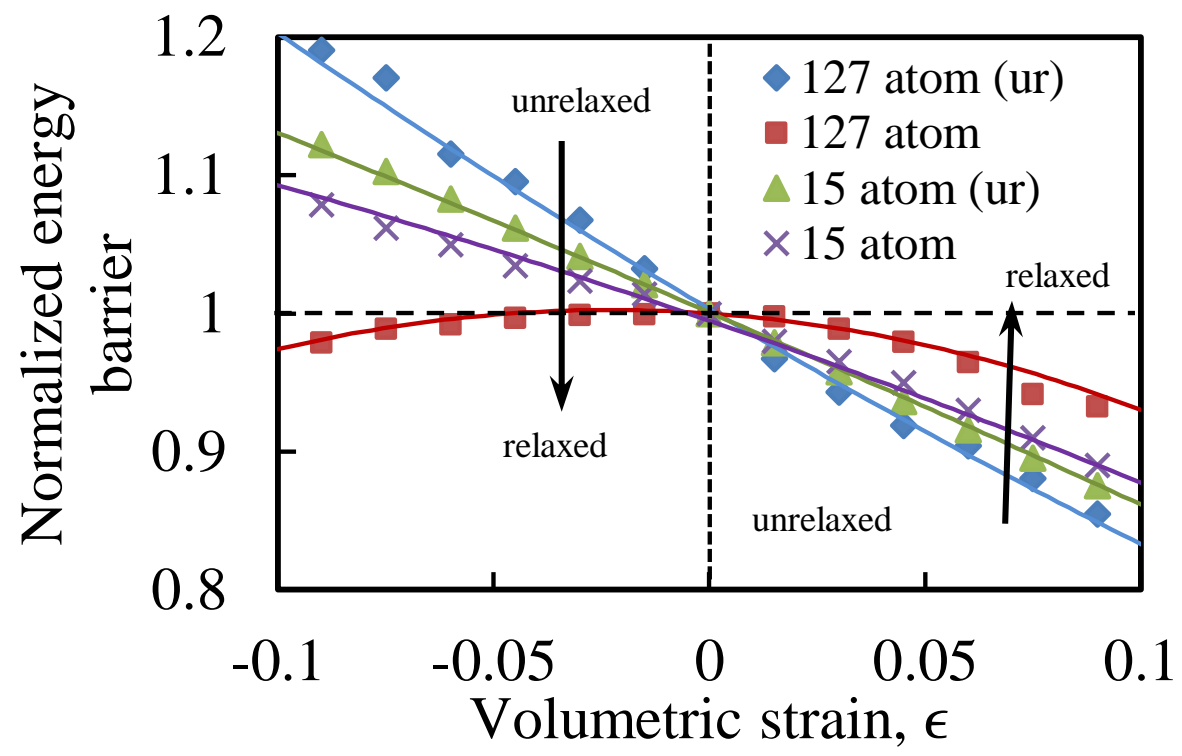

Figure 8: Normalized migration energy barrier for Re in W. The blue and red lines show migration energies for 127-atom supercells under relaxed and unrelaxed conditions, respectively. The cyan and green lines show migration energies for 15-atom supercells under relaxed and unrelaxed conditions, respectively. The effect of local relaxation on migration energy barrier at constant volume is marked by the arrows.

only marginally with relaxation. Conversely, for the $4 a_{0} \times 4 a_{0} \times 4 a_{0}$ supercell, the behavior shifts from linear (unrelaxed) to quadratic (relaxed). On the basis of these results, we take the relaxed calculations for the 127-atom system as the definitive ones and proceed to discuss them.

The overall strain dependent behavior for these calculations is well captured by the following 
quadratic fit:

$$
\Delta \bar{H}_{\mathrm{m}}=-4.77 \epsilon^{2}-0.219 \epsilon+1.0
$$

It is worth noting that the migration energy barrier decreases under both compression and tension in W. This is at odds with similar calculations in $\mathrm{Al}$ (Ho et al., 2007), where, using Kohn-Sham DFT for a 32-atom supercell and orbital-free DFT for a 108-atom cell, $\Delta H_{\mathrm{m}}$ is seen to increase under compression and decrease under tension. This would be consistent with the 15-atom cell calculations reported in Fig. 8, and, therefore, we argue that the linear behavior may be an artifact of cell scale limitations.

\subsubsection{Dependence with shear deformation}

As shown schematically in Fig. 9, there are two distinct paths under simple shear with two distinct migration energies for a substitutional Re atom to migrate to one of the nearest-neighbor sites. For

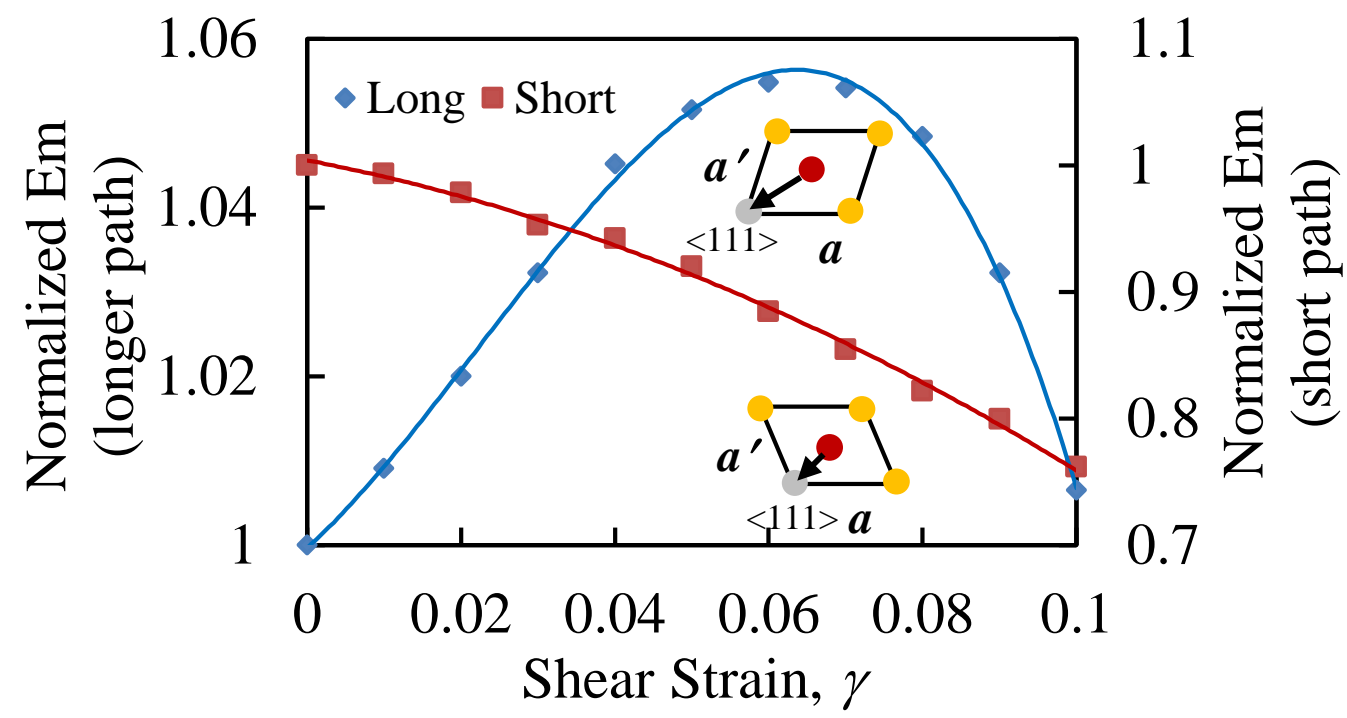

Figure 9: Path-dependent normalized migration energy barrier under simple shear deformation. The schematic image shows the corresponding paths.

the longer path, the migration energy gradually increases with applied deformation and reaches a maximum at around $\gamma=0.065$, where $H_{m}$ is $5.5 \%$ higher than the unstrained value. Thereon it decreases sharply until recovering the unstrained value at approximately $10 \%$ shear deformation. For its part, the migration energy for the shorter path always decreases with applied shear deformation. Thus, under shear deformation, a Re atom always prefer the shortest path, regardless of the amount of deformation applied. The polynomial fits that best represent these dependencies are:

$$
\begin{aligned}
\Delta \bar{H}_{\mathrm{m}}^{\text {long }} & =-235 \gamma^{3}+16 \gamma^{2}+0.82 \gamma+1.0 \\
\Delta \bar{H}_{\mathrm{m}}^{\text {short }} & =-12.8 \gamma^{2}-1.16 \gamma+1.0,
\end{aligned}
$$

For pure shear, the migration energy follows a pattern very similar to the simple shear case, as shown in Fig. 10. In this case, the lattice vectors of the deformed lattice are $\boldsymbol{a}_{1}=\left(a^{\prime}, 0,0\right) ; \boldsymbol{a}_{2}=$ $(a \sin \theta, a \cos \theta, 0) ; \boldsymbol{a}_{3}=(0,0, a)$, where, $a^{\prime}$ and $a$ are the deformed and undeformed lattice parameter, and $a^{\prime}=\frac{a}{\sqrt{\cos \theta}}$. The $3^{\text {rd }}$-degree least squares fit to the variation in migration energy with shear strain $\gamma$ is:

$$
\Delta \bar{H}_{\mathrm{m}}=-522 \gamma^{3}+32.8 \gamma^{2}+0.77 \gamma+1.0
$$




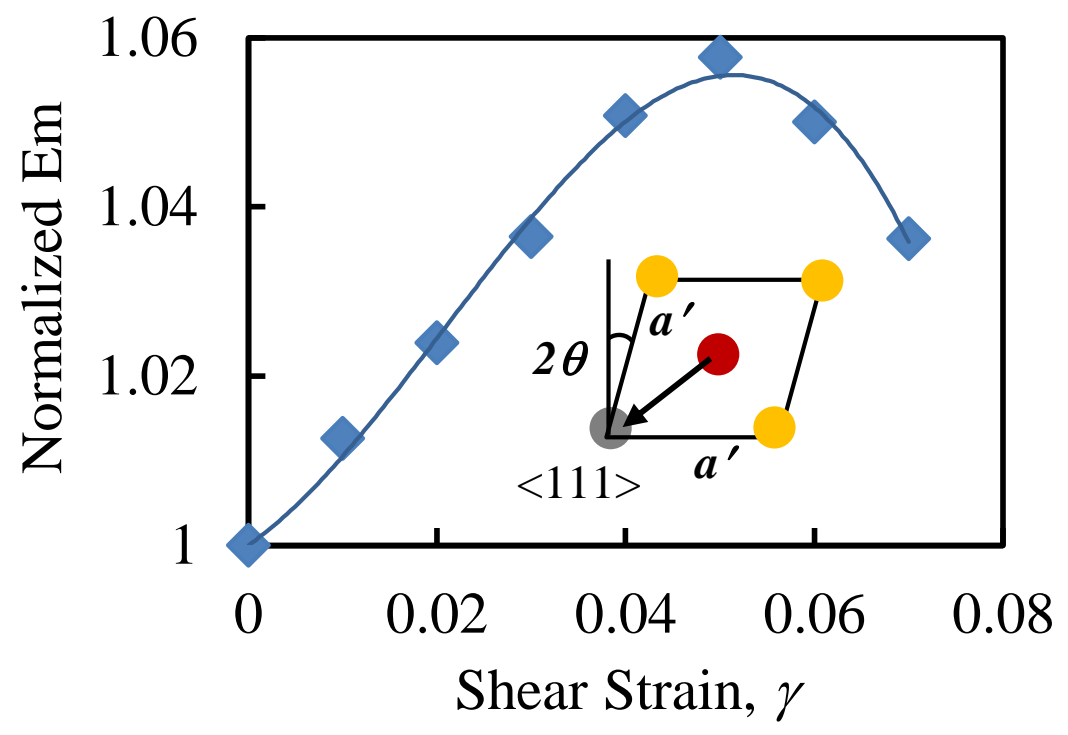

Figure 10: Normalized migration energy barrier under simple shear deformation along the longer path, as indicated in the schematic.

\section{Dislocation-Solute Interactions}

The impact of Re solute atoms on dislocation segments can be decomposed into elastic and inelastic contributions. The elastic contribution originates from treating solute atoms as point sources of dilatation while the inelastic one stems from the binding of electronic and mechanical nature of Re atoms and dislocation cores. Next, we analyze both contributions in detail.

\subsection{Elastic interaction energy due to Re substitution}

The long-range elastic interaction energy arises from the effective size difference between the solute atom Re and the solvent $\mathrm{W}$ atoms, and is usually characterized using isotropic linear elasticity, where the solute atom is treated as a point of dilatation inserted in an elastic matrix. Consequently, it is a source only of hydrostatic stress, $p$, and therefore couples only to the diagonal components of the dislocation stress field. The interaction energy between a dislocation and a solute atom is given by (Argon, 2004; Leyson et al., 2010):

$$
\Delta H_{\mathrm{int}}=p \Delta V_{f}=B \epsilon \Delta V_{f}
$$

where $\epsilon$ is the volumetric strain, $B$ is the bulk modulus, and $\Delta V_{f}$ is the formation volume of a substitutional Re atom. To compute $\Delta V_{f}$, we perform DFT calculations with supercells containing one Re atom in $\mathrm{W}$ matrix and relaxing the system to zero pressure. We considered five different supercell sizes and the results were seen to converge for a 128-atom supercell, as illustrated in Fig. 11. We find a converged value of $\Delta V=-1.4 \AA^{3}$, which indicates that a Re solute atom occupies a smaller volume than a matrix $\mathrm{W}$ atom. This results in local tensile stresses in the lattice upon insertion of the Re atom. Thus, from Eqn. 15, the strain dependence of the interaction energy can be expressed as a linear function of $\epsilon$ :

$$
\Delta H_{\mathrm{int}}=-2.69 \epsilon[\mathrm{eV}]
$$

The stress tensor in spherical coordinates due to a solute atom can be obtained by treating it as 


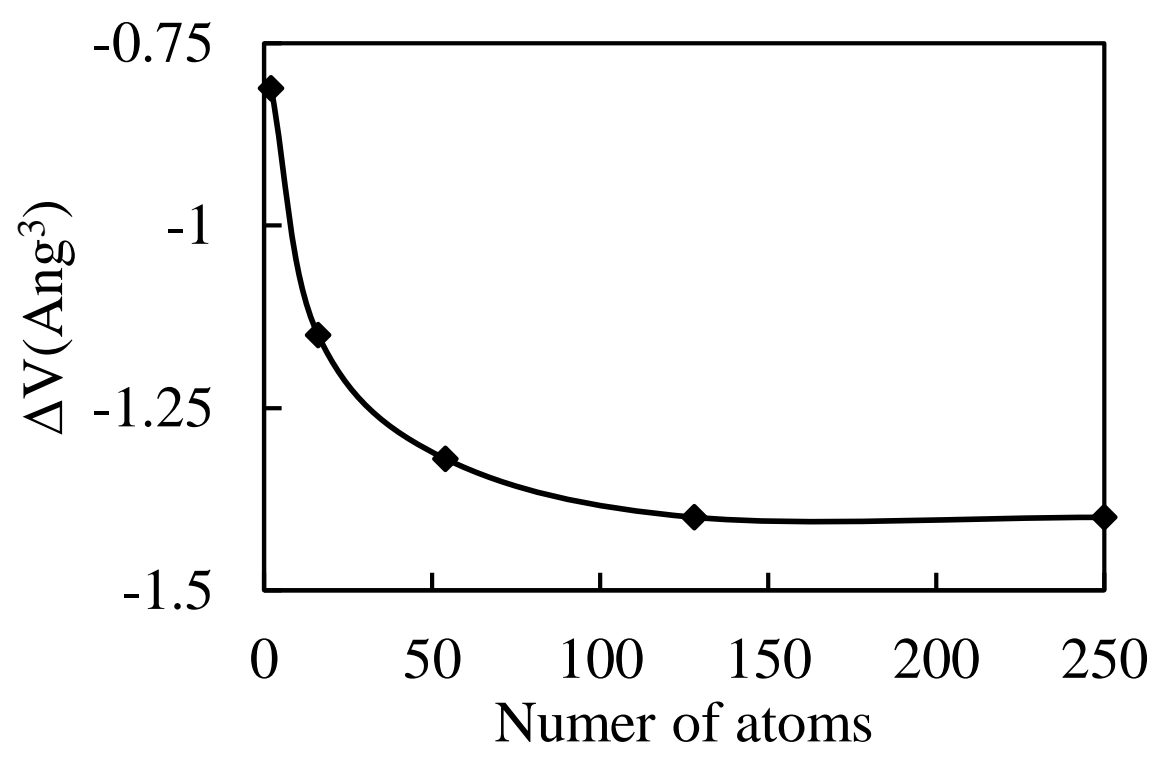

Figure 11: Dependence of the solute formation volume $\Delta V_{f}$ as a function of the number of atoms in the supercell calculations.

an Eshelby inclusion (Teodosiu, 1982):

$$
\begin{aligned}
\sigma_{r r} & =-\frac{4 \mu \delta}{r^{3}} \\
\sigma_{\theta \theta} & =\sigma_{\phi \phi}=\sigma_{r r} / 2
\end{aligned}
$$

where $\delta$ can be written as:

$$
\delta=\frac{\Delta V_{f}(1+\nu)}{12 \pi(1-\nu)}
$$

Because $\delta$ multiplies the shear modulus $\mu$ directly in Eqs. 16, even a value of a few percent may induce large stresses, particularly in the vicinity of the inclusion. From Eqs. 16 and 17, the stress due to the substitutional Re atom becomes:

$$
\sigma_{r r}=\frac{38.9}{r^{3}}
$$

which gives the stress in GPa when $r$ is in $\AA$. The above function is plotted in Figure 12 and displays the classical singularity of linear elasticity. As the figure shows, at distances $r>7.0 \AA$, the stress falls below $100 \mathrm{MPa}$.

\subsection{Solute-dislocation binding energy}

Next, we study the short range interaction between a Re atom and $1 / 2\langle 111\rangle$ edge and screw dislocations cores. This interaction is intrinsically inelastic and can no longer be treated with linear elasticity. In such cases, the interaction energy is equivalent to the binding energy $E_{b}$, representing either attraction $\left(E_{b}<0\right)$ or repulsion $\left(E_{b}>0\right)$. The binding energy represents the excess energy released (or absorbed) by making the bound objects infinitely separated and can be expressed for a dislocation and a Re atom as:

$$
H_{b}=E_{\operatorname{Re}+\S}\left(r=r_{0}\right)-E_{\operatorname{Re}+\S}(r=\infty)
$$




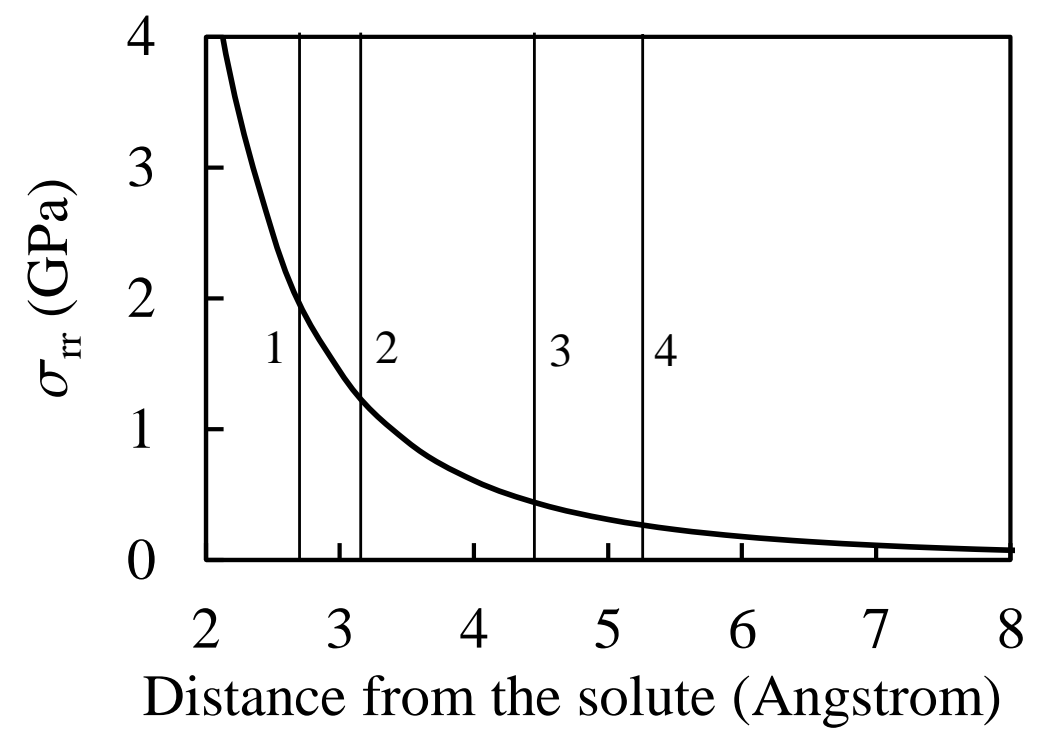

Figure 12: Radial dependence of $\sigma_{r r}$ as computed in this work using DFT calculations. Each of the labeled vertical lines represents a nearest-neighbor distance. At the first nearest neighbor shell $\sigma_{r r}$ has a value of $1.9 \mathrm{GPa}$ (tensile).

where the $\S$ symbol represents a dislocation core, and $r_{0}$ is the core radius, within which elasticity theory cannot be used. To compute $E_{b}$, we use configurations of balanced screw and edge dislocation dipoles that preserve the periodicity of the computational cell. To that end, we construct crystallites of size $n_{x} \times n_{y} \times n_{z}$ multiples of the bcc lattice vectors $\frac{1}{2}[111] \times[1 \overline{1} 0] \times[11 \overline{2}]$. The unit cell vectors are $a_{x}=a_{0} \frac{\sqrt{3}}{2}=2.716 \AA, a_{y}=a_{0} \sqrt{2}=4.434 \AA$, and $a_{z}=a_{0} \sqrt{6}=7.681 \AA$.

\subsubsection{Edge dislocation dipole}

To create an edge dislocation dipole, a computational cell with $n_{x}=14, n_{y}=8$, and $n_{z}=1$ unit cells containing 672 atoms is constructed and a set of three $\{111\}$ planes is removed over the central region of the box. This effectively results in a $1 / 2[111](1 \overline{1} 0)$ edge dislocation. The undeformed configuration obtained after this transformation contains 648 atoms and is shown in Fig. 13a. In such a small dipole construction, the removal of atoms to generate the dipole leads to high distortions along the [111] direction. To mitigate this effect, the box is relaxed to zero pressure along that direction, so that the resulting effective lattice constant becomes $2.9826 \AA$, down from the nominal value of $3.1336 \AA$ (cf. Table 1). The relaxed configuration is shown in Fig. 13b.

To compute the binding energies, the Re atom is placed at different locations on two distinct (110) planes above and below the dislocation core. The plane above the core is situated in the compressive region of the displacement field — denoted by ' $c$ ' in Fig.13b — whereas the plane below - denoted by ' $d$ '- belongs to the tensile or dilatational region. The binding energies for each region as a function of distance to the core are given in Fig. 14. The figure clearly shows that the converged binding energy in the compressive region $(0.45 \mathrm{eV})$ is significantly larger than its counterpart in the tensile region $(0.07 \mathrm{eV})$. This would favor the accumulation of Re atoms in the compressive strain field zone of the edge dislocation, consistent with the results shown in Fig. 12 and the sign of $\Delta V_{f}$ found in the previous section.

We note that, rather than a single isolated solute, the present calculations entail a periodic train of solute atoms separated by a distance of $7.68 \AA$ and interacting elastically. However, as shown in Fig. 12, this interaction can be neglected for this separation distance. 


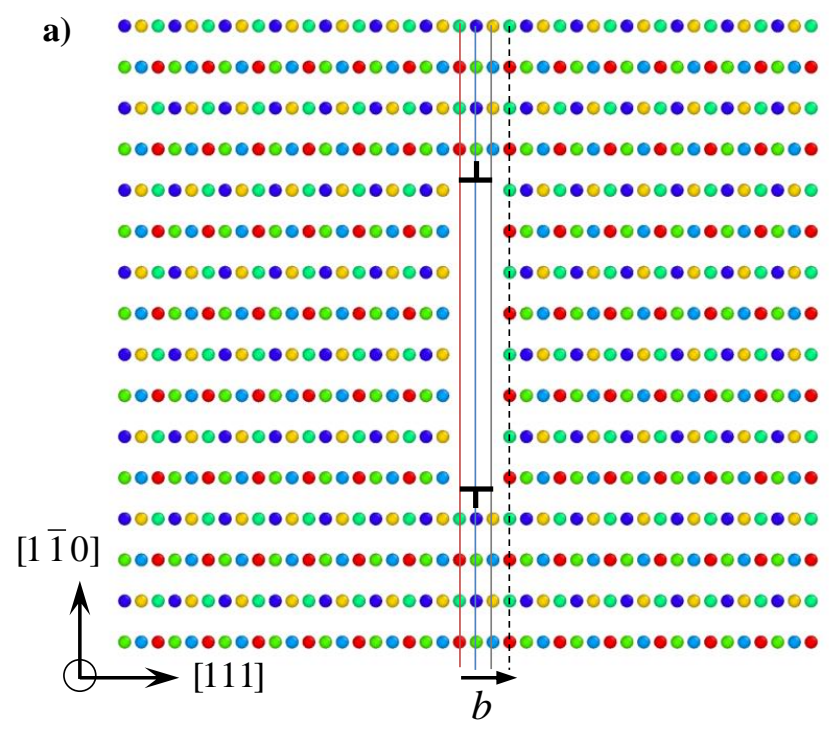

b) $\bullet 00 \bullet 00 \bullet 00 \bullet 00 \bullet 00 \bullet 00 \bullet 00 \bullet 00 \bullet 00 \bullet 00 \bullet 00 \bullet 00 \bullet 00 \bullet 00$

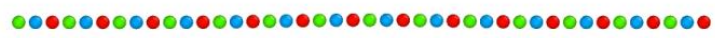

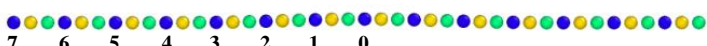
c

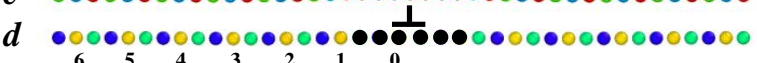

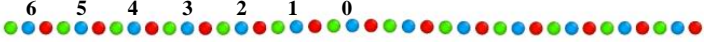

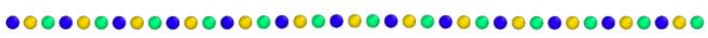

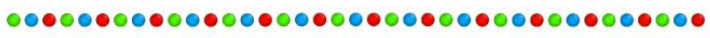

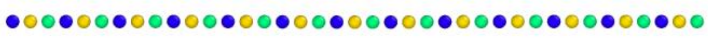

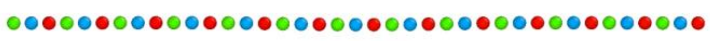

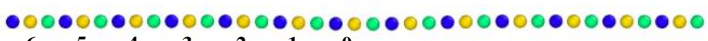

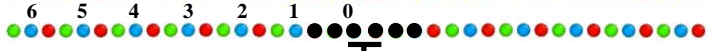

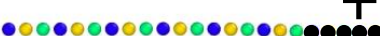

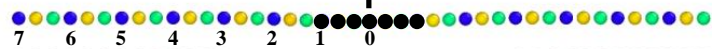

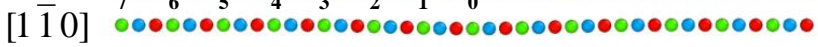

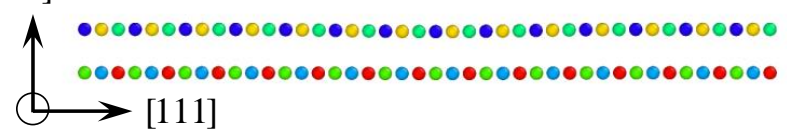

Figure 13: $[11 \overline{2}]$ view of (a) the unrelaxed and (b) relaxed edge dislocation dipole configurations. The three (111) planes removed to generate the edge dislocation dipole, as well as the Burgers vector $\boldsymbol{b}$, are shown in the lower panel. Figure 13(b) also shows the demarcation of the atomic positions where Re atoms were inserted for calculating binding

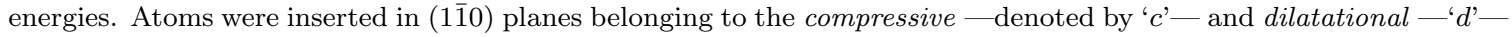
regions of the edge dislocation displacement field. The dislocation core is highlighted in black, symbolizing atoms that do not display a local bcc structure.

\subsubsection{Screw dislocation dipole}

The screw dislocation structure is constructed in super cells with $n_{x}=6, n_{y}=7$, and $n_{z}=1$ containing 252 atoms and by imposing the Volterra displacement fields (Cai et al., 2003): $u_{z}=$ $u_{[111]}=\frac{b \theta}{2 \pi}=\frac{b}{2 \pi} \arctan \frac{x}{y}, u_{x}=u_{[1 \overline{1} 0]}=0, u_{y}=u_{[11 \overline{2}]}=0$. This structure is relaxed and the resulting dislocation cores analyzed using differential displacement maps. The results are shown in Fig. 15, where the dislocation cores are seen to display a compact structure, in accordance with recent studies (Romaner et al., 2010; Samolyuk et al., 2013). 


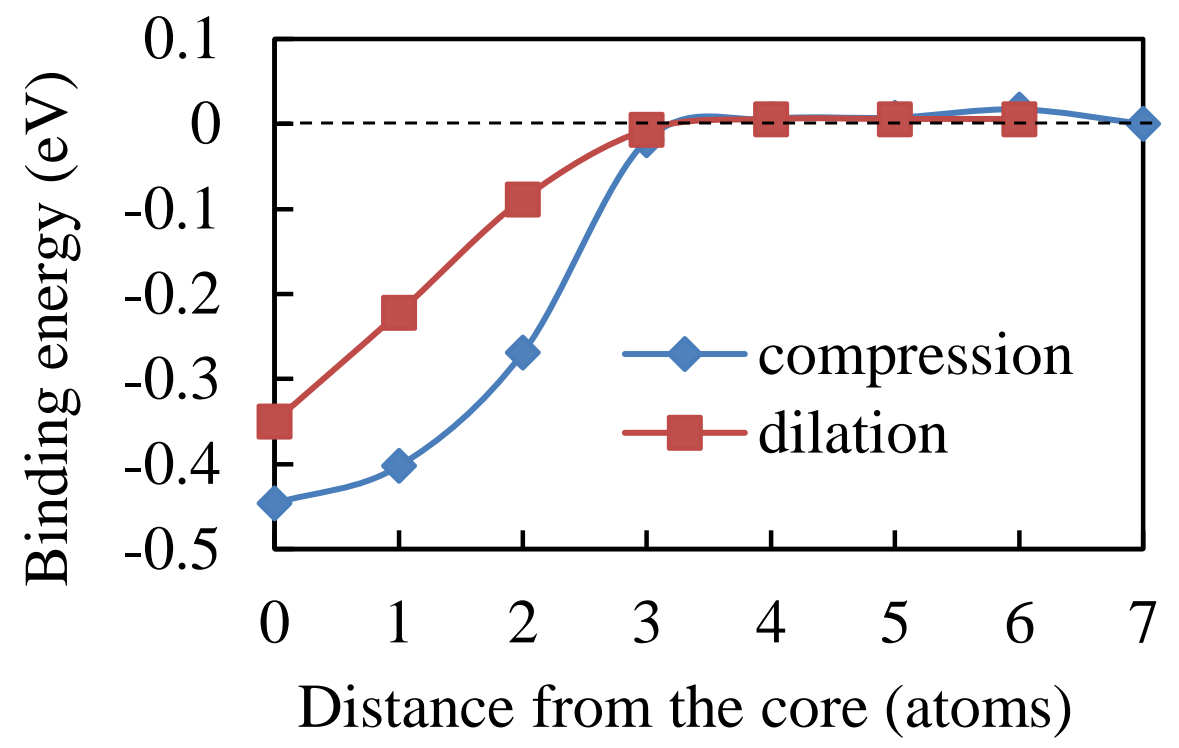

Figure 14: Binding energy of a Re substitutional atom to an edge dislocation core in different locations labeled according to Fig. 13.

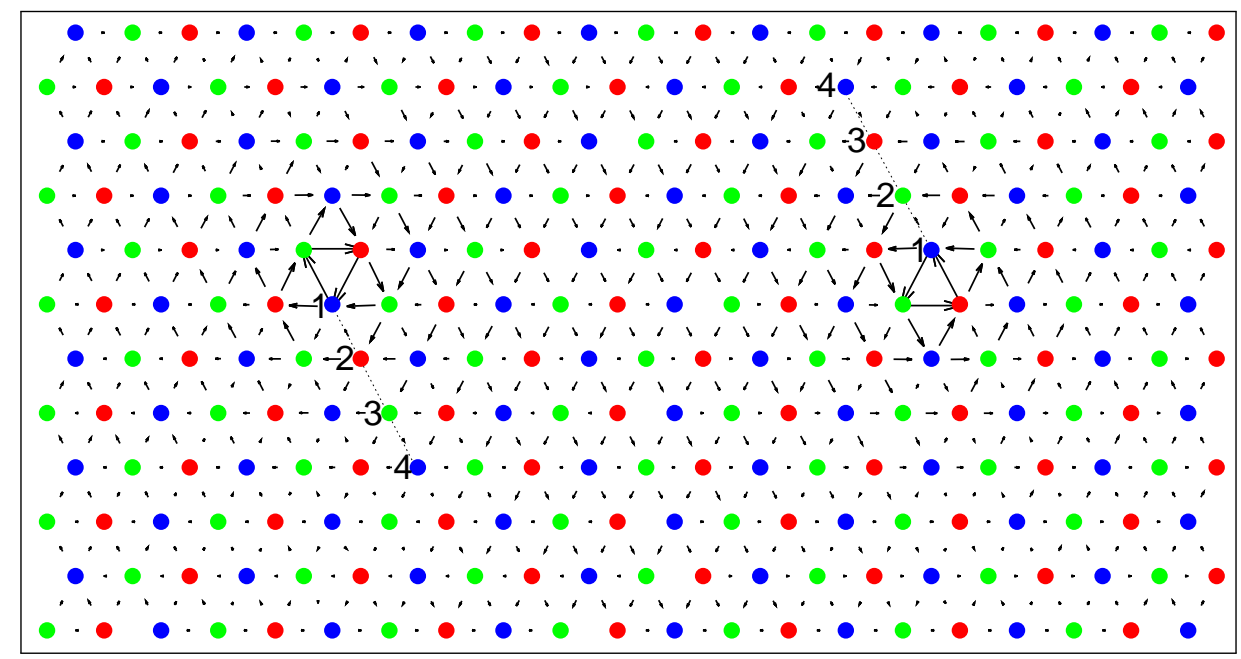

Figure 15: [111] view of the differential displacement map showing the compact structure of the screw dislocation core in W. Atomic sites labeled numerically near the left core indicate the positions where Re atoms were inserted to compute the binding energies, cf. Fig. 16.

The atomic positions labeled ' 1 ' through ' 4 ' in the figure mark where Re atoms have been inserted for computing $E_{b}$, and are aligned along one of the sixfold degenerate directions radiating away from the dislocation core. To balance the dipole construction, this construction is replicated for both cores ensuring the preservation of the threefold core symmetry. The energetics of the solute atom at these locations are given in Figure 16, showing the binding energy to be approximately $0.25 \mathrm{eV}$. Thus, from an energetics perspective, a Re atom is more favorably drawn to an edge dislocation core than to a screw dislocation one. This is not surprising given the weak volumetric component of the screw dislocation displacement fields. 


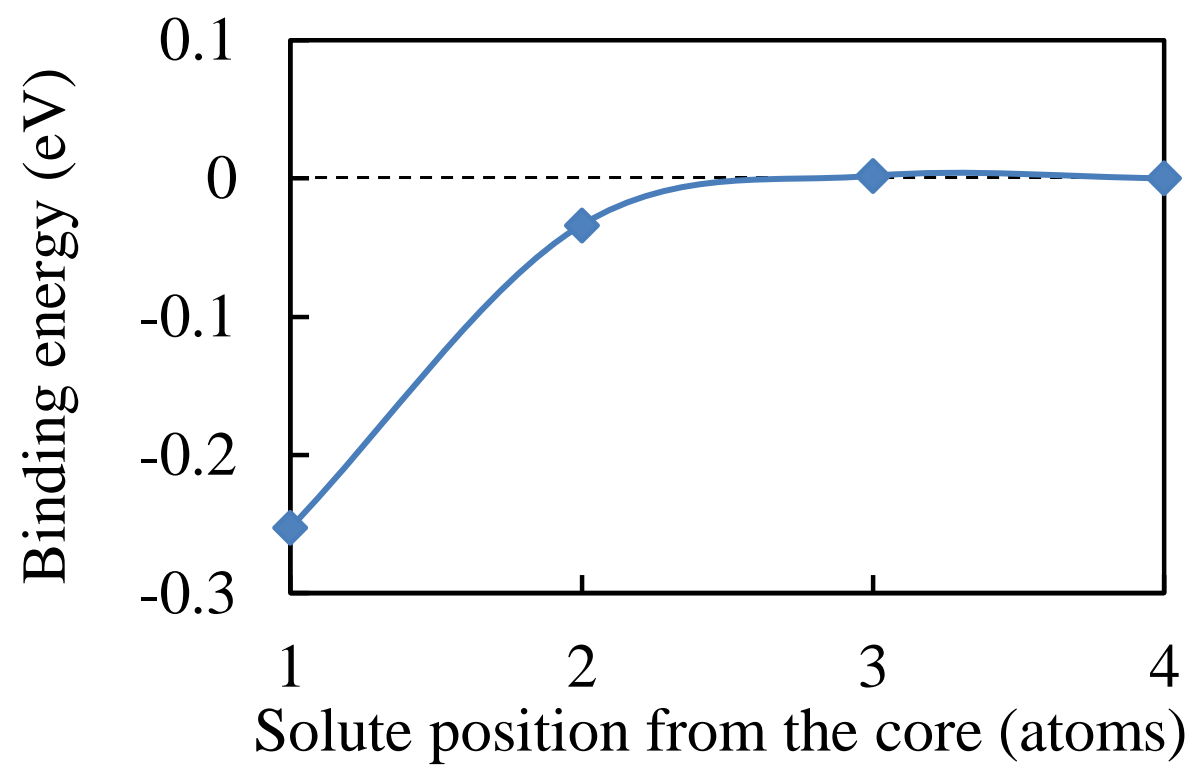

Figure 16: Binding energy of a Re substitutional atom to a screw dislocation core for different locations labeled according to Fig. 15 .

As above, these calculations include the interaction energies of a periodic array of solutes. In this case, the separation distance is of only $2.716 \AA$, so that the elastic interaction is not as low as in the edge dislocation case. This fact must be kept in mind when using these numbers.

\section{Stress dependent solute kinetics}

As pointed out in Section 2.1, the ultimate purpose of the calculations presented here is to furnish stress-dependent kinetic coefficients for solute diffusion in W-Re alloys. The jump rate in eq. 1 contains exponential terms characterizing solute diffusion by a vacancy mechanism in the presence of dislocation stress fields. The enthalpies in those exponential terms are known to display the following dependence on stress:

$$
\Delta H=\Delta E-W_{m}=\Delta E-\sigma: \mathbf{V}^{a}
$$

with $V_{i j}^{a}=\frac{\partial E}{\partial \sigma_{i j}}$. Thus, we decompose the activation volume tensor into diagonal and off-diagonal components in terms of the volumetric and shear strains:

$$
V_{i j}^{a}=\left\{\begin{array}{c}
\frac{1}{3 B} \frac{\partial E}{\partial \epsilon}, i=j \\
\frac{1}{\mu} \frac{\partial E}{\partial \gamma}, i \neq j
\end{array}\right.
$$


where $E(\epsilon)$ and $E(\gamma)$ have been characterized in Section 3. Then, the mechanical work $W_{m}=\sigma: \mathbf{V}^{a}$ can be reduced to the following expression:

$$
\begin{array}{r}
W_{m}=\frac{1}{3 B} \frac{\partial E}{\partial \epsilon}\left(\sigma_{11}+\sigma_{22}+\sigma_{22}\right)+ \\
+\frac{2}{\mu} \frac{\partial E}{\partial \gamma}\left(\sigma_{23}+\sigma_{13}+\sigma_{12}\right)= \\
=\frac{p}{B} \frac{\partial E}{\partial \epsilon}+\frac{2}{\mu} \frac{\partial E}{\partial \gamma}\left(\sigma_{23}+\sigma_{13}+\sigma_{12}\right)
\end{array}
$$

If one now decomposes the jump frequency $J$ into a vacancy concentration, $J_{v}$, a migration, $J_{m}$, and a binding, $J_{b}$, term:

$$
J=J_{0} J_{v} J_{m} J_{b}=J_{0} \exp \left(-\frac{\Delta H_{f}+\Delta H_{m}+\Delta H_{b}}{k T}\right)
$$

we can then characterize each contribution separately. We note that $E_{b}=0$ if $r<r_{0}$, with $r_{0}$ defined as in eq. 18. In particular, the corresponding terms for eq. 19 are:

1. From eq. $9, \partial E_{\mathrm{v}} / \partial \epsilon=-65.8 \epsilon+11.6$

2. From eq. $10, \partial E_{\mathrm{v}} / \partial \gamma=-33145 \gamma^{2}+593.6 \gamma-8.71$

3. From eq. $11, \partial E_{\mathrm{m}} / \partial \epsilon=-15.0 \epsilon-0.34$

4. From eq. 13,

$$
\partial E_{\mathrm{m}} / \partial \gamma=\left\{\begin{array}{l}
-1107 \gamma^{2}+50.2 \gamma+1.28, \text { long jump } \\
-40.2 \gamma-1.82, \text { short jump }
\end{array}\right.
$$

\section{Concluding remarks}

In conclusion, we have carried out first-principles calculations of stress-dependent energetics of Re-atom transport in bulk W, as well as the energetics of the interactions between Re atoms and $1 / 2\langle 111\rangle$ screw and edge dislocations in W. We find that volumetric deformation affects vacancy formation energy and migration energy barrier differently. The stress induced by substitutional Re atom in $\mathrm{W}$ leads to stresses in the range of MPa up to the fourth nearest neighbors from the defect center. For the edge dislocation core, the substitution is favored in the compressive side of the core, suggesting a possible asymmetric distribution of the defect population near the core. For screw dislocations, the biding energy of a Re atom is much higher than the tensile region in the edge core, but lower than the compressive region in the edge core.

\section{Acknowledgments}

This work was carried out under the auspices of the U.S. Department of Energy by Lawrence Livermore National Laboratory under Contract DE-AC52-07NA27344. MZH is very grateful to

Prof. Kaushik Bhattacharya and the PSAAP-Caltech program. JM acknowledges support from DOE/OFES Early Career Program. 
Argon, A. S., 2004. Strengthening Mechanisms in Crystal Plasticity. Oxford Univ. Press.

Cai, W., Bulatov, V. V., Chang, J., Li, J., Yip, S., 2003. Periodic image effects in dislocation modelling. Philosophical Magazine 83 (5), 539-567.

Carling, K., Wahnström, G., Mattsson, T. R., Mattsson, A. E., Sandberg, N., Grimvall, G., Oct 2000. Vacancies in metals: From first-principles calculations to experimental data. Phys. Rev. Lett. 85, 3862-3865.

Ceperley, D. M., Alder, B., 1980. Ground state of the electron gas by a stochastic method. Physical Review Letters 45 (7), 566.

Delczeg, L., Delczeg-Czirjak, E. K., Johansson, B., Vitos, L., Nov 2009. Assessing common density functional approximations for the $a b$ initio description of monovacancies in metals. Phys. Rev. B $80,205121$.

Fu, C.-C., Torre, J. D., Willaime, F., Bocquet, J.-L., Barbu, A., 2005. Multiscale modelling of defect kinetics in irradiated iron. Nature Materials 4, 68-74.

Giusepponi, S., Celino, M., 2013. The ideal tensile strength of tungsten and tungsten alloys by first-principles calculations. Journal of Nuclear Materials 435 (13), $52-55$.

Hinterberg, J., Zacherle, T., De Souza, R. A., May 2013. Activation volume tensor for oxygenvacancy migration in strained $\mathrm{ceO}_{2}$ electrolytes. Phys. Rev. Lett. 110, 205901.

Ho, G., Ong, M. T., Caspersen, K. J., Carter, E. A., 2007. Energetics and kinetics of vacancy diffusion and aggregation in shocked aluminium via orbital-free density functional theory. Phys. Chem. Chem. Phys. 9, 4951-4966.

Korzhavyi, P. A., Abrikosov, I. A., Johansson, B., Ruban, A. V., Skriver, H. L., May 1999. Firstprinciples calculations of the vacancy formation energy in transition and noble metals. Phys. Rev. B 59, 11693-11703.

Lassner, E., Schubert, W.-D., 1999. Tungsten: properties, chemistry, technology of the elements, alloys, and chemical compounds. Springer.

Leyson, G. P. M., Curtin, W. A., Hector Jr, L. G., Woodward, C. F., 2010. Quantitative prediction of solute strengthening in aluminium alloys. Nature materials 9 (9), 750-755.

Li, H., Wurster, S., Motz, C., Romaner, L., Ambrosch-Draxl, C., Pippan, R., 2012. Dislocation-core symmetry and slip planes in tungsten alloys: Ab initio calculations and microcantilever bending experiments. Acta Materialia 60 (2), 748-758.

Mattsson, A. E., Armiento, R., Schultz, P. A., Mattsson, T. R., May 2006. Nonequivalence of the generalized gradient approximations pbe and pw91. Phys. Rev. B 73, 195123.

Mattsson, T. R., Mattsson, A. E., Dec 2002. Calculating the vacancy formation energy in metals: Pt, pd, and mo. Phys. Rev. B 66, 214110.

Nazarov, R., Hickel, T., Neugebauer, J., Apr 2012. Vacancy formation energies in fcc metals: Influence of exchange-correlation functionals and correction schemes. Phys. Rev. B 85, 144118.

Romaner, L., Ambrosch-Draxl, C., Pippan, R., May 2010. Effect of rhenium on the dislocation core structure in tungsten. Phys. Rev. Lett. 104, 195503.

Samolyuk, G. D., Osetsky, Y., Stoller, R. E., 2013. The influence of transition metal solutes on the dislocation core structure and values of the peierls stress and barrier in tungsten. Journal of Physics: Condensed Matter 25 (2), 025403. 
Satta, A., Willaime, F., de Gironcoli, S., May 1998. Vacancy self-diffusion parameters in tungsten: Finite electron-temperature lda calculations. Phys. Rev. B 57, 11184-11192.

Schultz, H., Ehrhart, P., 1991. Atomic Defects in Metals. Springer, Berlin.

Söderlind, P., Yang, L. H., Moriarty, J. A., Wills, J. M., Jan 2000. First-principles formation energies of monovacancies in bcc transition metals. Phys. Rev. B 61, 2579-2586.

Soler, J. M., Artacho, E., Gale, J. D., García, A., Junquera, J., Ordejón, P., Sánchez-Portal, D., 2002. The siesta method for ab initio order- $\mathrm{n}$ materials simulation. Journal of Physics: Condensed Matter 14 (11), 2745.

Teodosiu, C., 1982. Elastic models of crystal defects. Springer-Verlag.

Troullier, N., Martins, J. L., Jan 1991. Efficient pseudopotentials for plane-wave calculations. Phys. Rev. B 43, 1993-2006.

Voter, A. F., 2007. Radiation Effects in Solids. Vol. 235. Springer.

Watson, R., Davenport, J., Weinert, M., Fernando, G., 1988. Structural stability of rhenium as a function of lattice compression: Theory. Phys. Rev. B: Condens. Matter;(United States) 38 (11).

Weinberger, C. R., Tucker, G. J., Foiles, S. M., Feb 2013. Peierls potential of screw dislocations in bcc transition metals: Predictions from density functional theory. Phys. Rev. B 87, 054114.

Wierzbowska, M., Fleszar, A., May 2011. Density functional theory calculations for a single re impurity in silicon. Phys. Rev. B 83, 184418.

Wurster, S., Gludovatz, B., Hoffmann, A., Pippan, R., 2011. Fracture behaviour of tungstenvanadium and tungsten-tantalum alloys and composites. Journal of Nuclear Materials 413 (3), $166-176$.

Wurster, S., Gludovatz, B., Pippan, R., 2010. High temperature fracture experiments on tungstenrhenium alloys. International Journal of Refractory Metals and Hard Materials 28 (6), 692-697.

Zhou, H.-B., Jin, S., Zhang, Y., Lu, G.-H., Liu, F., 2012. Anisotropic strain enhanced hydrogen solubility in bcc metals: The independence on the sign of strain. Physical Review Letters 109 (13), 135502 .

Zinkle, S. J., Ghoniem, N. M., 2011. Prospects for accelerated development of high performance structural materials. Journal of Nuclear Materials 417 (1), 2-8. 